\title{
A Comparison Between National Scheme for the Acoustic Classification of Dwellings in Europe and in the U.S.
}

\author{
Umberto Berardi \\ Department of Architectural Science, Faculty of Engineering and Architectural Science, Ryerson University, 350 \\ Victoria St, Toronto, ON M5B 2K3, Canada

\begin{abstract}
Birgit Rasmussen
Danish Building Research Institute, Aalborg University (AAU-CPH), A.C. Meyers Vnge 15, 2450 Copenhagen SV, Denmark
\end{abstract}

(Received 20 April 2014; accepted 18 February 2015)

\begin{abstract}
The classification of dwellings according to different building performances has been proposed through many schemes worldwide in recent years. The general idea behind these schemes relates to the positive impact a higher label, and thus a better performance, should have. In particular, focusing on sound insulation performance, national schemes for sound classification of dwellings have been developed in several European countries. These schemes define acoustic classes according to different levels of sound insulation. Due to the lack of coordination among countries, a significant diversity in terms of descriptors, number of classes, and class intervals occurred between national schemes. However, a proposal "acoustic classification scheme for dwellings" has been developed recently in the European COST Action TU0901 with 32 member countries. This proposal has been accepted as an ISO work item. This paper compares sound classification schemes in Europe with the current situation in the United States. Economic evaluations related to the technological choices necessary to achieve different sound classification classes are also discussed. The hope is that a common sound classification scheme may facilitate exchanging experiences about constructions fulfilling different classes, reducing trade barriers, and finally increasing the sound insulation of dwellings.
\end{abstract}

\section{INTRODUCTION}

Recent research has proven how sound insulation deeply correlates with productivity, higher learning outcomes, and concentration. $^{1-3}$ However, beyond annoyance or decreased productivity, poor acoustical environments also affect health. The World Health Organization has repeatedly linked the population's exposure to environmental noise with adverse health effects and has established a relationship between traffic noise and an increased risk of cardiovascular diseases. ${ }^{4,5}$ At the same time, an emerging body of research has linked the exposure to environmental noise with sleep disturbance, cognitive impairment in children, and Tinnitus, among other human health concerns. ${ }^{4,6}$ All of these studies show that the indoor acoustic performance deeply matters for the quality of the built environment.

The majority of countries in Europe have a long tradition of regulatory sound insulation requirements for dwellings, going back to the $1950 \mathrm{~s}$ in some cases. ${ }^{7,8}$ The purpose of these regulations is the protection of health. However, the fulfilment of these requirements does not ensure satisfactory conditions, and the protection is typically insufficient for sensitive persons or in the case of loud neighbours. For this reason, classification schemes have been introduced in several countries to specify higher levels of acoustical comfort. The first classification schemes for dwellings were implemented in the early 1990s. ${ }^{8}$ A scheme generally defines a number of classes according to a certain interval of some acoustic indicators that are used to reflect different levels of acoustic comfort.
Findings from comparative studies of regulatory sound insulation requirements and sound classification schemes in Europe show that sound insulation descriptors, regulatory requirements, and classification schemes have a high degree of diversity. ${ }^{9-11}$ These studies have concluded that harmonization is needed to facilitate the exchange of data and experience among countries and to reduce trade barriers.

This paper considers that sound insulation requirements are a national issue that cannot be made homogenous worldwide. However, the comparison of the ongoing experience towards a harmonized European classification scheme with the situation in the U.S. would help building stakeholders to familiarize with acoustic classes fitting local needs and conditions.

\section{CLASSIFICATION SCHEMES IN EUROPE}

\subsection{Existing Sound Classification Schemes in Europe}

A sound classification scheme could be defined as a set of a minimum of three classes with different sound insulation performance levels. Using this definition, classification schemes for dwellings exist, at present, in ten countries in Europe. ${ }^{8}$ Sound classification schemes indicate different quality classes to meet different needs of activities and quietness. ${ }^{10}$ They have generally been developed as technical standards, but sometimes they are already referred to in national laws.

Acoustic class information could be considered as an analogy to energy labelling or other labelling systems. In fact, any 